Revue européenne des sciences sociales

European Journal of Social Sciences

XLII-129 | 2004

La sociologie durkheimienne : tradition et actualité

\title{
Esthétique et rationalité du choix des prénoms
}

\section{Pierre Demeulenaere}

\section{OpenEdition}

\section{Journals}

Édition électronique

URL : http://journals.openedition.org/ress/383

DOI : $10.4000 /$ ress.383

ISSN : 1663-4446

\section{Éditeur}

Librairie Droz

\section{Édition imprimée}

Date de publication : 1 mars 2004

Pagination : 105-116

ISBN : 2-600-00941-8

ISSN : 0048-8046

Référence électronique

Pierre Demeulenaere, "Esthétique et rationalité du choix des prénoms 》, Revue européenne des sciences sociales [En ligne], XLII-129 | 2004, mis en ligne le 05 novembre 2009, consulté le 02 mai 2019. URL : http://journals.openedition.org/ress/383 ; DOI : 10.4000/ress.383 
Pierre DEMEULENAERE

\section{ESTHÉTIQUE ET RATIONALITÉ DU CHOIX DES PRÉNOMS}

Dans leurs travaux sur les prénoms, $\mathrm{Ph}$. Besnard et $\mathrm{G}$. Desplanques évoquent à plusieurs reprises la dimension «esthétique» du choix de ceux-ci ${ }^{1}$. Le remarquable modèle de choix « rationnel $»^{2}$ des prénoms qu'ils mettent en avant est ainsi une contribution originale à l'étude de la formation des goûts. Il n'est en effet pas habituel du point de vue d'une théorie standard du choix rationnel d'y inclure l'analyse des préférences esthétiques jugées généralement «irrationnelles », ou en tout cas étrangères à la sphère de la rationalité (relevant, pour employer le langage des économistes, des préférences révélées, ou données). Dans cet article, je me propose de 1) préciser la signification exacte de cette imputation de rationalité au choix des prénoms, en essayant en particulier d'indiquer les limites du modèle proposé par rapport aux données statistiques relatives à ces choix; 2) de déterminer la spécificité de cette dimension «esthétique» du choix des prénoms, qui permet de contribuer à une sociologie de l'esthétique en général. Il est en effet important de ne pas cantonner les études de sociologie de l'esthétique aux études de sociologie de l'art, et la contribution de Besnard et Desplanques est à cet égard fort éclairante. Elle montre l'importance des sentiments esthétiques dans la vie quotidienne, par-delà le registre institutionnel plus ou moins clair des «beauxarts».

Pour atteindre ces deux objectifs, je m'appuierai directement sur les travaux de Besnard et Desplanques: à la fois les données statistiques qu'ils livrent et les commentaires qu'ils proposent pour l'élucidation de ces données. En effet, ils mettent en œuvre une théorisation de ces choix et de ces préférences, et c'est cette théorisation qui sera ici discutée, dans la mesure où elle a des implications fortes pour l'élucidation du rapport entre goût individuel et goût collectif.

\section{CHOIX DES PRÉNOMS ET MODÈLE DE RATIONALITÉ}

A quoi correspond donc cette rationalité du choix des prénoms? Voilà ce qu'en disent les deux auteurs:

Besnard P. et Desplanques G. (1999).

Besnard P. et Desplanques G. (2003, p. 11). Dans la suite de cet article les indications de page renvoient, sauf mention autre, à cet ouvrage. 
Le choix des prénoms est généralement le fruit d'une stratégie rationnelle; c'est l'addition de ces choix individuels qui produit des effets inattendus, et cela d'autant plus que l'on est dans l'ignorance des choix d'autrui.

Depuis que les contraintes et les modèles traditionnels (souci de marquer une identité familiale, religieuse ou locale) ont disparu, la motivation fondamentale des parents, ou au moins de la grande majorité d'entre eux, n'a rien de mystérieux; elle est à la fois consciente et rationnelle, même si elle procède de deux préoccupations contradictoires. D'un côté, les parents veulent individualiser leur enfant; c'est leur enfant et non un autre. Il faut donc éviter un prénom trop répandu, divulgué, voire vulgaire. Mais, d'un autre côté, ils savent bien qu'un prénom excessivement rare court le risque d'être excentrique, extravagant, à la limite intolérable pour son porteur. C'est dans cet entre-deux, dans cet intervalle entre le commun et l'excentrique, que le choix va s'effectuer.

De là vient cette affinité élective entre le prénom et la mode. Car le phénomène de mode naît précisément de cette tension entre l'originalité et le conformisme. Pour entrer dans la ronde de la mode, un produit nouveau doit être différent du produit antérieur banalisé et usé. Il doit permettre à son acquéreur de se distinguer des autres ou, plus exactement, de marquer ses distances avec ceux dont il entend se distinguer, mais, en même temps, d'affirmer sa ressemblance avec ceux auxquels il s'identifie ou dont il souhaite se rapprocher. (p. 11)

Rappelons que dans la tradition des sciences sociales, la notion de rationalité s'applique généralement au choix des moyens vis-à-vis de certaines fins, ce qu'il est convenu d'appeler la rationalité instrumentale. Dans le cadre ici retenu, la rationalité correspond bien à cela: le choix des prénoms est un moyen permettant de parvenir à certains buts. Pourtant, implicitement, la théorie de la rationalité ici décrite se situe semble-t-il en fait à deux niveaux: d'une part, les parents, confrontés à la nécessité du conformisme et de l'individualisation, assument ces deux motifs, qui s'imposent à eux, mais qui sont donc aussi d'un certain point de vue eux-mêmes choisis rationnellement (ce qui n'est pas évident, comme j'essaierai de l'indiquer). Par la suite, ayant ces motifs, ils choisissent de manière efficace (mais avec des effets inattendus) les prénoms qui permettent de les satisfaire.

Les motifs sont donc clairement définis et donnés, pour tout acteur: un mélange de souci d'originalité et de conformisme, lequel est générateur du phénomène de mode. D'où viennent ces soucis, et comment s'articulent-ils? On peut remarquer à cet égard une asymétrie: d'un côté, le souci d'individualisation fait aujourd'hui (dans une société précisément individualiste) largement partie de la fonction du prénom, qui est de permettre de reconnaître et de désigner un individu, ce qui conduit à éviter que tout le monde ait le même prénom. Ce motif peut être plus ou moins net mais on peut considérer qu'il est imposé par la logique de la situation: il n'est pas du tout problématique et dérive d'un besoin pratique. Il serait absurde que tout le monde ait le même prénom, et à partir de là, naît une tendance à la multiplication de leur registre.

En revanche, le souci de conformisme est moins évident: pour en rendre compte, les auteurs évoquent les risques liés au port du prénom très rare (dans une explication un peu redondante: on explique le conformisme par la crainte d'être extravagant). Toutefois, de fait, il existe des parents qui, délibérément, ne choisissent pas des prénoms déjà établis. Juridiquement, la question a été posée de savoir si le choix des prénoms doit se faire à partir d'un stock préalable préconstitué, clairement balisé, ou s'il peut y avoir une «invention» de nouveaux prénoms. A cet égard, les évolutions récentes tendent à indiquer que d'une part les prénoms 
anciens tendent à revenir sur le devant de la scène, qu'ils sont accompagnés de nouveaux prénoms d'origine étrangère (essentiellement anglo-saxonne et latine), mais qu'il y a aussi des inventions nettes (au moins dans l'usage) du type «Ludivine», «Titouan» ou «Mathis» (p. 214, p. 287 et p. 234), désormais admises par la loi en France.

Dès lors, l'idée de rationalité ne permet en fait en rien de savoir si le choix du prénom sera plus ou moins conformiste, toute la théorie de Besnard et Desplanques conduisant à mettre précisément en évidence la diversité des types de choix à cet égard: le choix peut être en effet «excentrique», «précurseur», «pionnier», «dans le vent», «conformiste», «à la traîne» ou «démodé» (par exemple Amandine, p. 95). Autrement dit, plutôt qu'une théorie unifiée de la rationalité du choix, qui permettrait de prédire un certain type uniforme de sélection de prénom, au regard de sa fréquence plus ou moins élevée, on a plutôt la description d'une diversité de choix «typiques » ${ }^{3}$, qui sont à la limite opposés (précurseur ou à la traîne). Par ailleurs ces choix typiques manifestement différents peuvent être rapportés soit à des états d'esprit psychologiques (goût ou non pour l'innovation), soit à des situations sociales hétérogènes (je reviendrai plus loin sur ce point important: la position sociale permet-elle de prédire la diversité des types de choix effectués?).

Ce qu'ont donc mis en évidence, de manière remarquable, Besnard et Desplanques, c'est la tendance macro-sociologique à l'essor et au déclin des prénoms, appuyée sur des tendances micro-sociologiques à l'innovation d'un côté et au conformisme de l'autre. Il s'agit donc de tendances contraires, dont l'existence de chacune se conçoit aisément: à vrai dire, tout choix est nécessairement (mais tout est relatif dans la mesure où les chiffres sont eux-mêmes relatifs) soit conformiste soit non-conformiste (innovant ou à la traîne), ces polarités opposées admettant une pluralité de positions intermédiaires.

La forme typique (la plus fréquente) de la courbe d'évolution des prénoms vient de ce contraste entre les attitudes innovantes et les attitudes conformistes qui sont reliées chronologiquement: l'innovation précède logiquement le conformisme; et lui succède aussi puisque les innovants ne sauraient se porter vers les prénoms déjà établis, et donnent lieu, par le lancement de nouveaux prénoms, à de nouvelles possibilités de conformisme (les anciennes n'étant pas toujours complètement abolies). Le conformisme est plus ou moins fort et donne lieu à des séquences différenciées.

Mais on n'a pas ici réellement une théorie du choix rationnel individuel, on a plutôt la constatation de choix essentiellement divergents, qui sont par ailleurs plus ou moins fréquents dans une population donnée. Il est intéressant d'ailleurs de noter que si la courbe des prénoms est fréquemment (mais pas toujours) en forme de cloche, dans la réalité il y a une grande diversité de la longueur des séquences temporelles correspondant à un nombre donné de prénoms attribués par année, ce qui augmente la diversité des types de choix, puisque ceux-ci peuvent être ainsi plus ou moins longtemps choisis comme précurseurs, comme confor-

Il est vrai qu'un choix qualifié par le sociologue, ex post, de conformiste, ne correspond pas nécessairement à une intention conformiste, ex ante, de l'acteur. On peut néanmoins convenir que les résultats typiques peuvent correspondre aussi en gros à des intentions typiques qui leur sont corrélées. 
mistes ou comme démodés. Autrement dit, même s'il y a des pics «conformistes » dans le choix d'un prénom donné, la longueur des différentes séquences de choix (très variable en fonction des différents prénoms) peut faire que les nombres globaux de choix relevant de chaque type atteignent des scores qui éloignent d'un contraste vif entre «innovation» et «conformisme».

Un candidat plus resserré et plus précis au modèle de choix rationnel serait alors le suivant: tout acteur doit faire un choix à la fois relativement innovant et relativement conformiste (hypothèses de base liées à la logique de la situation, et désignant ces motifs comme des moyens permettant d'atteindre des objectifs partagés). A partir de là, chaque acteur choisit donc un prénom relativement peu fréquent, mais non marginal, sans savoir que les autres acteurs font de même: il atteint alors un pic, ce qui tend à détourner les successeurs (doués de la même structure de préférences) de ce prénom devenu désormais trop courant. Ici, on aurait bien une figure univoque d'un acteur rationnel dont les choix pourraient tour à tour se porter vers un prénom puis se détourner de lui en fonction de la connaissance des résultats des choix similaires effectués par les autres acteurs. La rationalité impliquerait l'ajustement temporel au nombre global de prénoms, fluctuant du fait de décisions rationnelles antérieures faites en situation d'information limitée (les acteurs choisissant un prénom ne savent pas que les autres vont faire le même choix - sauf s'ils lisent Besnard et Desplanques).

Ce modèle est extrêmement séduisant (et il correspond sans doute à ce qu'ont voulu mettre en évidence Besnard et Desplanques pour rendre compte de la forme de la courbe); il souffre cependant de plusieurs défauts à notre sens majeurs.

Tout d'abord, il ne permet pas de rendre compte de la diversité des choix effectifs, de l'importance des choix pionniers comme de l'importance des choix «à la traîne », sans parler du phénomène considérable des choix «classiques » se portant vers des prénoms relativement à l'abri des phénomènes de mode (type «Pierre»). L'idée d'un comportement rationnel implique, si on veut lui donner un contenu précis et univoque, une certaine uniformité de choix (dans le cas standard, le choix des moyens par rapport à une fin). Or ce que mettent en évidence les auteurs c'est plutôt une diversité de ces choix, le comportement typique «choisir un prénom peu répandu mais pas rare » n'étant qu'un des éléments d'une gamme plus large, même s'il est souvent le plus fréquent.

Ensuite, ce modèle aurait pour conséquence normative de considérer les choix précurseurs, à la traîne ou classiques (ou purement excentriques: choix rares sans lendemains) comme «irrationnels », ce qui est franchement absurde. Il y a toujours une dimension normative dans l'imputation de rationalité, mais on voit mal pourquoi un choix précurseur, classique ou à la traîne pourrait être jugé irrationnel. Il est intéressant de ce point de vue de remarquer que les auteurs de cette recherche donnent des «conseils» dans le guide La cote des prénoms en 2004 (destiné au grand public et pouvant partiellement fonctionner comme prophétie auto-réalisatrice). Ces conseils dérivent en gros du modèle de rationalité évoqué précédemment: il «faut» choisir un prénom qui ne soit ni trop fréquent ni trop rare. Lorsque l'on regarde les nombreux sites internet consacrés au choix des prénoms (qui citent très largement les travaux de Besnard et Desplanques sans toujours en mentionner les auteurs), on y trouve clairement un souci normatif de choisir le «bon» prénom, de «ne pas se tromper». Ici comme ailleurs, la moyenne et les tendances de comportement sont interprétées en «norme», ce qui ne va évidemment pas de soi. 
Il y a trois autres phénomènes considérables qui ne sont pas expliqués par ce modèle du choix rationnel dans la version précise que nous venons d'évoquer (identité de comportement) ou même dans une version large, qui insisterait seulement sur des soucis différenciées de conformisme et d'innovation (même si, à notre sens, ces seuls soucis ne permettent pas de justifier un modèle qui puisse être dit réellement «rationnel»: car cette notion implique une certaine uniformité des choix soutenue d'une manière ou d'une autre normativement).

Premièrement, comme il vient d'être dit, le choix «classique» (du type Pierre, Jean ou Marie) échappe largement à cette dialectique innovation/conformisme. Le registre limité de ce type de prénom ne saurait masquer leur importance (Pierre est ainsi le deuxième prénom le plus porté en France).

Deuxièmement, ces deux variables innovation/conformisme ne permettent pas d'expliquer la diversité des choix entre milieux «populaires» et milieux «bourgeois » (dans le cadre de cet article, et à la suite de Besnard et Desplanques, on utilisera ces deux catégories comme emblématiques de polarités tendancielles, même si, bien évidemment, l'analyse des prénoms comme l'analyse sociologique en général invite à des distinctions beaucoup plus précises et nuancées). On peut dire à ce sujet plusieurs choses: il y a des prénoms clairement «mixtes » (aussi fréquents en milieu populaire qu'en milieu bourgeois), de type «Alexandre»:

\begin{abstract}
Choisi en France pour près d'un nouveau-né sur 100 pendant une bonne partie du XIX siècle, Alexandre décline doucement depuis 1890 et ne disparaît pas complètement durant sa période de purgatoire. Sa nouvelle carrière, d'abord lente à se dessiner, l'a conduit au sommet: un garçon sur 40, et cela dans tous les milieux sociaux, presque toutes les régions, et durant onze ans (p. 91)
\end{abstract}

Au contraire, il y a des prénoms qui témoignent clairement d'une polarité opposée des choix, le plus célèbre en France étant peut-être Kevin, «prénom nettement populaire, très peu connu en milieu bourgeois » (p. 201) à l'opposé d'un Eudes si clairement aristocratique. Il faut noter toutefois qu'il est impossible de trouver des propriétés intrinsèques (tirées de la forme seule des prénoms) qui permettent d'expliquer cette polarité: car d'un côté, comme nous venons de le voir, un même prénom peut être adopté aussi bien en milieu populaire qu'en milieu bourgeois, mais, surtout, des formes extrêmement proches d'un prénom peuvent donner lieu à des spécialisations sociales opposées: ainsi Jocelyn et Jocelyne. De surcroît, un même prénom peut, au cours de sa carrière dans le temps, passer d'un milieu à l'autre, et cela dans les deux sens. Il y a en effet une diffusion verticale de l'innovation des milieux bourgeois avant-gardistes vers les milieux plus populaires; mais en sens inverse des prénoms autrefois populaires peuvent réapparaître dans des milieux bourgeois innovants qui le destinent à une nouvelle carrière:

Beaucoup de prénoms qui reviennent aujourd'hui au goût du jour sont ceux que l'on
considérait, au début du XX $X^{\mathrm{e}}$ siècle, comme convenant aux domestiques parce qu'ils
étaient parvenus au terme de leur trajectoire sociale. Il faut aller dans les campagnes les
plus reculées, y lire sur le monument aux morts les prénoms des paysans (nés à la fin du
XIX ${ }^{\mathrm{e}}$ siècle) qui ont péri dans la Grande guerre. On y trouve un florilège des prénoms qui
montent ou qui vont percer. (p. 328 )

Ce dernier phénomène peut permettre d'inclure la revivification bourgeoise de prénoms populaires au cycle de l'innovation et de la diffusion hiérarchique à 
partir d'une indication supplémentaire: il s'agit de montrer que certaines positions sociales incitent davantage à l'innovation, tout simplement car celle-ci y est davantage accessible, par les connaissances et l'information dont disposent les acteurs dans la situation qui est la leur.

On a là un raisonnement sociologique très fréquent ${ }^{4}$ : on explique les motifs d'une catégorie d'acteurs, par opposition à ceux d'une autre, par le fait que ces motifs sont plus accessibles aux uns qu'aux autres dans la situation où ils se trouvent. Par exemple, il est plus facile d'avoir une multiplicité de partenaires sexuels dans une grande ville que dans un village, ce qui permet d' "expliquer» que dans les grandes villes il y ait davantage de personnes qui ont de très nombreux partenaires sexuels. Mais cette «explication » n’explique précisément pas la «volonté » elle-même d'avoir de nombreux partenaires sexuels qui est éventuellement une donnée caractéristique de certaines personnes, simplement favorisée dans un cas et réfrénée dans l'autre; il est ainsi possible, bien sûr, qu'une majorité de personnes vivant dans la grande ville ne souhaite pas avoir un grand nombre de partenaires sexuels. Mais on peut tout de même «expliquer» cette propension à la multiplicité des partenaires par les opportunités de la grande ville. Dans le cas de la tendance à l'innovation dans le choix des prénoms, on peut donc déduire la progression des prénoms des couches bourgeoises vers les couches populaires du fait que, par leur position, les premières sont davantage en situation d'innover que les secondes (car elles auraient un accès plus aisé à l'information permettant l'innovation: ce sont ainsi les professions du spectacle et de l'information qui ont la propension la plus forte à l'adoption de prénoms nouveaux, cf. p. 329-331). Par la suite, la visibilité donnée par ces couches avant-gardistes à certains prénoms leur permettraient d'être adoptés par ceux que leur position oriente moins facilement vers les recherches innovantes.

Ce schéma est néanmoins fortement concurrencé par un autre phénomène:

\begin{abstract}
Certaines innovations sont adoptées d'emblée en milieu populaire sans avoir été testées auparavant dans les milieux bourgeois, notamment les prénoms d'origine étrangère (ou de forme étrangère). C'est le cas par exemple d'Anthony - choix pionnier de 1970 à 1978 - ou de Kevin - précurseur de 1969 à 1978 et pionnier jusqu'en 1984 (et qui deviendra le prénom le plus donné en France de 1989 à 1994). Sur l'ensemble des prénoms, le flux vertical continue d'être à l'œuvre dans les années 70 et 80 comme le prouvent les écarts encore importants entre les catégories sociales. Mais, à cette diffusion verticale se superpose une diffusion segmentée où les flux horizontaux, internes à chaque milieu social, prennent de plus en plus de part. Le resserrement de la hiérarchie temporelle des goûts a ainsi pour contrepartie un accroissement et un durcissement de leur stratification dans l'espace social's.
\end{abstract}

Il y a donc clairement un phénomène d'hétérogénéité sociale des goûts en matière de prénoms qui, s'il n'est pas systématique, est néanmoins réel et significatif. Il ne se réduit donc pas à la dialectique innovation/conformisme, et à son interprétation éventuelle en termes de choix rationnel.

Enfin, un troisième domaine où cette dialectique innovation/conformisme est insuffisante pour rendre compte des données relatives au choix du prénom est

J'ai parlé à ce sujet de raisons de possibilité dans Demeulenaere (2003).

5 Besnard et Desplanque (1999, p. 104). Ce diagnostic est réitéré plusieurs fois (2003, p. 340). 
celui du style même des prénoms sélectionnés, et de leur essor plus ou moins grand dans tel ou tel milieu ou dans l'ensemble de la population. Nous avons déjà vu une différenciation des goûts en fonction de leur caractère «classique» ou «à la mode». Il y a de plus une différenciation entre milieux. Mais il y a aussi clairement des «styles » de prénoms qui s'imposent dans des périodes données, en sorte que les prénoms émergents, ou ceux qui sont à l'apogée, partagent un «air de famille » (ce qui signifie inversement qu'un certain style de prénoms possibles ne se développe pas du tout). Ainsi pour les prénoms étrangers: il faut déjà noter le phénomène significatif et massif qui est que, dans l'ensemble, les Français tendent à choisir pour leurs enfants des prénoms «français». Il y a toutefois, essentiellement en milieu populaire, une tendance à l'adoption de prénoms étrangers, mais tirés de deux sources principales: anglo-saxonne et celtique, ou latine. Il n'y a quasiment pas de prénoms asiatiques, africains ou arabes qui soient donnés aux rejetons de familles d'origine française, alors que des populations ayant ces origines étrangères sont nombreuses en France et sont même françaises le plus souvent. Cette absence ne peut s'expliquer uniquement par des difficultés de prononciation qui ne concernent en rien un «Mohamed» ou un «Kim». Par ailleurs Besnard et Desplanques mettent bien en évidence une stylistique unifiant des catégories de prénoms, en fonction de leur terminaison par exemple (p. 55). Autrement dit, les prénoms anciens d'un passé révolu ne sont pas un réservoir d'où ressurgissent de manière aléatoire les nouveaux prénoms: il y a bien aussi une tendance à l'unification stylistique de ceux-ci, même si cette tendance peut être à terme contrecarrée si elle devient trop prégnante (innovation) (ibid.).

Il s'agit donc d'essayer de comprendre la logique de formation des préférences esthétiques en matière de prénom, en insistant à la fois sur le rôle essentiel de la dialectique innovation/ conformisme et sur les limites de celle-ci au regard des données observables. Il convient toutefois de préciser d'abord ce qui relève effectivement de cette dimension esthétique. Pour ce faire je m'appuierai sur des thèmes que j'ai essayé de développer de manière systématique à propos des formes stylistiques: la pluralité des sources de plaisir associées à un objet donné, l'existence de croyances et de présupposés dans l'appréhension des objets esthétiques, et la tendance rationalisatrice à l'acceptation de la pluralité stylistique ${ }^{6}$.

\section{LE SENTIMENT ESTHÉTIQUE ET LE CHOIX DES PRÉNOMS}

Lorsque les parents décident du prénom qu'ils vont donner à leurs enfants, ils délibèrent, et disent, le plus souvent, que tel ou tel prénom leur «plaît» ou non, et, à l'issue d'une confrontation des possibles, et des jugements attachés à chacun de ceux-ci, plus ou moins clairement formulés, ils s'orientent vers une décision qui a un caractère d'optimisation (subjective) compte tenu des préférences préalables (de toute sorte) qu'ils impliquent dans la comparaison. De tels engagements dans le choix des prénoms sont relativement déconcertants, car il est assez clair, comme le disent Besnard et Desplanques qu' «il n'y a pas de prénom beau ou laid en soi » (p. 13). Pourquoi?

\footnotetext{
$6 \quad$ Demeulenaere (2001).
} 
Cette formule peut être prise à deux niveaux: d'un point de vue descriptif, elle signifie qu'un même prénom peut faire alternativement l'objet de la faveur et de la défaveur, soit au cours du temps, soit en fonction des milieux sociaux. Mais on peut donner à cette affirmation une signification plus forte, qui est de souligner que les propriétés intrinsèques d'un prénom (leur forme sonore) ne permettent pas d'établir une distinction entre des «beaux » prénoms et des «prénoms laids », dans la mesure où il n'y a pas vraiment de marge de manœuvre dans la complexification de ces sonorités, comme cela existe en musique par exemple: un prénom a toujours une forme sonore assez sommaire qui ne permet pas réellement le développement d'une recherche esthétique poussée. De surcroît, le stock général des prénoms est assez limité (environ 2000, p. 10) et ce sont toujours les mêmes prénoms qui reviennent sur le devant de la scène, avec un nombre d'inventions pures assez limité. Il faut certes noter aussi l'introduction de prénoms étrangers, à l'origine spécialisée (anglo-saxonne, celtique et latine). La forte implication «esthétique» des membres de la vie sociale, lorsqu'ils ont à sélectionner un prénom, est donc déconcertante si l'on admet qu'il n'y a pas de prénoms beaux ou laids en soi. Elle demande à être décrite, et si possible expliquée, à partir d'un repérage de ce qu'est ici précisément cette dimension esthétique.

En effet, un prénom, comme tout objet susceptible d'être appréhendé d'un point de vue esthétique, a une réalité multidimensionnelle. Outre sa réalité sonore, il peut être associé à des «significations». Besnard et Desplanques s'opposent par exemple à «Gaylord» dont la signification leur paraît fâcheuse pour un enfant. Ces significations sont multiples et relèvent essentiellement de trois ordres:

- soit le sens concret associé à un prénom: Gaylord donc, mais Besnard et Desplanques évoquent aussi Fiacre ou Arcade, et associent au lancement d'une voiture le déclin de Mégane (non empêchée au Québec où la marque Renault n'est pas présente). Ces significations associées à un prénom font alors elles-mêmes l'objet de «préférences» qui ne sont pas directement d'ordre esthétique, mais relèvent précisément d'un souci d'éviter des interférences signifiantes trop fortes (et négatives) avec cette sphère esthétique, et qui peuvent néanmoins être intégrées au vocabulaire de ce qui «plaît» ou «déplaît» ${ }^{7}$. Ce sens peut aussi être rapporté à des figures emblématiques porteuses d'un prénom qui leur est alors associé et qui vont donner une orientation positive ou négative au prénom: la figure emblématique étant ici Adolphe, encore discrédité;

- soit l'appartenance communautaire et/ou religieuse: il est tout à fait improbable (on a là une certitude prédictive à peu près absolue) qu'un enfant issu de milieux juifs pratiquants soit prénommé Mohamed. Ces éléments sont évidemment toujours présents: mais lorsqu'un prénom illustre la volonté d'appartenance religieuse, ce choix cesse d'être esthétique à proprement parler (même si, là encore, une personne peut «aimer» des prénoms parce qu'ils contribuent à la solidarité communautaire qui a des résonances émotion-

Lorsque Besnard et Desplanques évoquent une prononciation française «disgracieuse» de prénoms d'origine étrangère, ils évoquent en fait le sens associé à cette prononcation, pour «SueEllen » par exemple (p. 48). 
nelles). Il a déjà été noté que les Français se portent essentiellement vers des prénoms français (qui intègrent toute la variété de leurs origines, romaines par exemple) mais aussi anglo-saxons et celtes, ou latins. On a là vraisemblablement une identification communautaire étendue, qui s'élargit dans certaines directions, évoquant des groupes qui sont d'une part présents dans la culture populaire, mais qui d'autre part ne sont pas associés à des populations immigrées globalement défavorisées. Là de même, il n’y a pas ici de considérations directement esthétiques (Mohamed n'est pas moins beau que Kevin), mais il y a transfert sur le choix qui plaît ou non d'autres éléments, d'ordre communautaire, qui eux-mêmes «plaisent» ou non: les gens n'ont généralement pas envie d'être défavorisés. Ces soucis communautaires peuvent donner lieu à des stratégies intelligibles à partir des difficultés qu'elles peuvent poser: tel couple mixte (arabe/français) qui ne souhaite donner la primauté à aucune des communautés, pourra s'orienter vers un prénom «latin» évoquant à la fois le Sud et le Nord, et, s'il a la forme «Enzo » par exemple, échappant à une connotation religieuse claire, tout en étant par ailleurs «dans le vent».

- enfin, le prénom évoque plus ou moins clairement des origines sociales: JeanHubert n'est pas Kevin, alors que, comme on l'a vu, Alexandre sépare de manière beaucoup moins claire les catégories sociales. Dès lors, les parents, consciemment (ou même inconsciemment, si l'on admet, comme Durkheim, l'existence d'un inconscient social ${ }^{8}$ ), peuvent associer à tel ou tel prénom telle ou telle situation sociale et vouloir éventuellement soit affirmer cette appartenance sociale (quelle qu'elle soit), soit la dissimuler ou la changer par l'intermédiaire du prénom. Ceci est favorisé par le fait que dans l'ensemble les Français reconnaissent assez clairement la catégorie sociale à laquelle ils appartiennent ${ }^{9}$. Tous ces motifs sont concevables et interviennent certainement dans le choix des prénoms: d'un côté il peut y avoir une recherche de «distinction» sociale; mais de l'autre il peut y avoir aussi souci de maintenir un prénom correspondant à la situation sociale concernée. On peut imaginer qu'il n'est pas évident, en milieu ouvrier, de prénommer son fils Eudes, ce qui paraîtrait «déplacé », bien qu'évidemment non impossible: il y a là un conformisme proprement social lié tendanciellement à tous les milieux, et qui est souligné par exemple par Schwartz quand il rapporte le fait que certains ouvriers cherchent à dissimuler l'acquisition de meubles de style non conformes aux habitudes et aux moyens de leur milieu ${ }^{10}$. Comme précédemment, ces dimensions non-esthétiques à proprement parler peuvent néanmoins être converties en langage esthétique à partir du moment où les prénoms symbolisent et représentent des situations sociales qui, par ailleurs, ellesmêmes, «plaisent» ou «déplaisent» dans la mesure où elles font l'objet d'un désir ou d'une aversion. Une difficulté générale de l'analyse est que peuvent être intégrés au registre de ce qui «plaît» ou «déplaît» à un individu des éléments qui peuvent ressortir au domaine de l'esthétique stricto sensu, mais aussi à des domaines tout à fait hétérogènes, comme l'appartenance commu-

\footnotetext{
Durkheim (1996).

Bréchon (2002).

o Schwartz (1990).
} 
nautaire ou le statut social qui produisent néanmoins des sentiments positifs ou négatifs assimilables dans le vocabulaire et peut-être dans l'expérience à des sentiments esthétiques.

Quelle est donc la spécificité esthétique du choix d'un prénom? Il y a vraisemblablement deux sources proprement esthétiques du choix du prénom.

La première tient à l'association d'idées privées, reliant tel prénom à des éléments qui font par ailleurs l'objet de «sentiments» positifs, sans que ceux-ci relèvent de l'appartenance sociale ou communautaire. Ces associations d'idée peuvent être strictement individuelles: une personne qui aura rencontré un magnifique Aurélien pourra reporter son admiration sur le prénom Aurélien. La Recherche du temps perdu est sans doute l'œuvre littéraire qui montre le mieux ces propensions imaginatives qui glorifient un nom ou un prénom de toutes sortes de qualités dérivées d'éléments variés qui lui sont liés de manière plus ou moins réelle ou fantasmatique. Ces associations d'idées d'ordre privé peuvent néanmoins acquérir une signification sociale lorsque des personnes situées dans des positions similaires sont confrontées simultanément à des objets qui typiquement leur plaisent par ailleurs en fonction de leur «culture»:

\footnotetext{
Ludivine est le prénom de l'hérö̈ne des gens de Mogador, livre, mais surtout feuilleton télévisé à succès, diffusé pour la première fois en 1972. Aussitôt naît ce prénom, hors d'usage auparavant. Il est rarissime de pouvoir ainsi repérer l'événement qui a lancé un prénom, même si Ludovic lui a peut-être préparé le terrain. Ludivine apparaît en même temps en toutes régions et dans toutes les catégories sociales, mais se développe mieux en milieu populaire.

Sa brusque poussée se tarit vite et Ludivine plafonne sans prénommer plus d'une fille sur 160. (pp. 214-215)
}

On peut comprendre alors que des prénoms se développent majoritairement dans certains milieux sociaux si ceux-ci sont dans une situation relativement étrangère à d'autres et sont confrontés simultanément aux mêmes événements pourvoyeurs d'associations d'idée suggestives favorisant l'adoption de prénoms qui «plaisent». A partir du moment où, pour une raison quelconque, un prénom, par exemple Ludivine, se diffuse, cette diffusion même tend alors à susciter une diffusion accrue ${ }^{11}$ par la visibilité du modèle qu'il représente, jusqu'à être ensuite limité, car les attitudes innovantes vont se porter vers un autre objet. Comme on l'a vu toutefois le rythme de ces succès et déclins est très variable, avec une tendance générale à l'accélération du cycle. Ces modèles de diffusion peuvent aussi favoriser le développement de formes sonores proches.

L'autre source éminemment esthétique du choix des prénoms est alors précisément la dialectique de l'innovation et de l'imitation, soit donc la rareté relative caractéristique du phénomène de la mode. Le phénomène de la mode met en scène clairement une dualité typique des comportements, suivant qu'ils sont pionniers ou suivistes, par-delà une unification par un modèle de choix rationnel instrumental ${ }^{12}$.

${ }^{11}$ On a là un phénomène analogue à la création d'une superstar dont le succès initial appelle et renforce le succès ultérieur (Menger, 2002).

12 Coleman (1990) essaie d'unifier les deux positions en un comportement rationnel, dépendant des ressources des acteurs, mais il y a clairement au bout du compte un contraste entre les innovateurs et les conformistes. 
Les travaux de Besnard et Desplanques permettent alors d'illustrer un phénomène remarquable qui dépasse largement le cadre des prénoms: un même objet peut plaire ou déplaire, suivant qu'il est ou nom à la mode. Ceci implique que l'objet est, en sus de ses propriétés spécifiques, appréhendé à travers des croyances, qui se portent ici vers la nouveauté et le fait qu'il soit favorisé par des «créateurs» dignes d'être imités. Mais ces croyances peuvent aussi favoriser, plutôt que la nouveauté, le caractère «classique » ou non d'un prénom. Ce caractère classique dérive d'une crainte du caractère éphémère des modes, et se porte vers des prénoms qui ont fait l'épreuve de leur résistance à ces effets de mode. Typiquement, en pays catholique, ces prénoms sont ceux que leur liaison initiale avec les très grands saints de la tradition a permis de maintenir constamment présents dans le temps sans être non plus spécialisés dans des milieux particuliers (type Pierre, Jean ou Marie).

On peut donc avoir une approche rationnelle du choix des prénoms, qui se déduit à la fois de l'analyse de la mode et des propriétés intrinsèques des prénoms (indépendamment des associations d'idée, conscientes ou inconscientes) dans une société individualiste relativement peu attachée au maintien de traditions prescriptives (même si celle-ci demeurent présentes). Elle correspond au fait que l'on ne peut pas considérer unilatéralement qu'il y ait des prénoms beaux ou laids en soi. Cette rationalité s'exprime alors à travers trois phénomènes significatifs, congruents avec ce que l'on observe par ailleurs au regard des formes stylistiques, qui sont le lieu à la fois d'une patrimonialisation générale des formes anciennes et d'un attachement à l'innovation et à la production de formes inédites:

Il y a une tendance à la pluralisation des prénoms, dont témoigne le fait qu'il y a de moins en moins de prénoms dominants (p. 308)

Cette pluralisation se fait notamment par une plus grande internationalisation (p. 320) (limitée néanmoins par la persistance d'identification communautaires, mais celles-ci sont élargies jusqu'à intégrer d'autres «cultures » rendues accessibles par les media, mais non associées à une situation défavorisée).

Elle se fait aussi par le retour latent de tous les prénoms du répertoire (favorisé par exemple normativement par les auteurs de la recherche, p. 50), qui sont repris et revivifiés après leur période de déclin. Les prénoms classiques ne sont qu’une variante de cette utilisation systématique de tous les prénoms appartenant au «patrimoine».

C'est donc, d'un certain point de vue, une autre rationalisation qui est ici à l'œuvre, et qui, comme dans le cas des formes stylistiques en général, contribue à faire un sort à la pluralité, laquelle conduit à intégrer les formes stylistiques du passé mais aussi à développer des formes nouvelles qui, dans le registre limité des prénoms, vont être trouvés à l'étranger ou dans la déclinaison nouvelle des prénoms anciens.

Université de Nancy 2

pierre.demeulenaere@noos.fr 


\section{RÉFÉRENCES BIBLIOGRAPHIQUES}

Besnard Ph. et Desplanques G. (1999), «Les catégories socioprofessionnelles à l'épreuve de la stratification temporelle des goûts », Revue française de sociologie, 60 (1), 97-109.

- (2003), La cote des prénoms en 2004, Paris, Balland.

Bréchon P. (éd.) (2000), Les valeurs des Français. Évolutions de 1980 à 2000, Paris, Armand Colin.

Coleman J. (1990), Foundations of Social Theory, Cambridge-London, The Belknap Press of Harvard University Press.

Demeulenaere P. (2001), Une théorie des sentiments esthétiques, Paris, Grasset.

- (2003), Les normes sociales. Entre accords et désaccords, Paris, PUF.

Durkheim É. [1898](1996), «Représentations individuelles et représentations collectives », in Sociologie et philosophie, Paris, PUF., 1-48.

Menger P.-M. (2002), Portrait de l'artiste en travailleur, Paris, Seuil.

Schwartz O. (1990), Le monde privé des ouvriers, Paris, PUF. 